\title{
RoboCup@Work: Competing for the Factory of the Future
}

\author{
Gerhard K. Kraetzschmar ${ }^{1}$, Nico Hochgeschwender ${ }^{1(凶)}$, Walter Nowak ${ }^{2}$, \\ Frederik Hegger $^{1}$, Sven Schneider ${ }^{1}$, Rhama Dwiputra ${ }^{1}$, Jakob Berghofer ${ }^{3}$, \\ and Rainer Bischoff ${ }^{3}$ \\ 1 Bonn-Rhein-Sieg University, Sankt Augustin, Germany \\ nico.hochgeschwender@h-brs.de \\ 2 Locomotec GmbH, Augsburg, Germany \\ 3 KUKA Laboratories GmbH, Augsburg, Germany
}

\begin{abstract}
Mobile manipulators are viewed as an essential component for making the factory of the future become a reality. RoboCup@Work is a competition designed by a group of researchers from the RoboCup community and focuses on the use of mobile manipulators and their integration with automation equipment for performing industrially-relevant tasks. The paper describes the design and implementation of the competition and the experiences made so far.
\end{abstract}

\section{Introduction}

For a long time, research on mobile robots and on industrial manipulators have been performed in separate communities. Recently, the robotics and automation industry is shifting its attention towards robotics scenarios involving the integration of mobility and manipulation, larger-scale integration of service robots and industrial robots, cohabitation of robots and humans, and cooperation of multiple robots and/or humans. All of these ideas are part of the factory of the future (FoF) (see also [10]), which is considered a foundation for conserving or reviving industrial competitiveness in the future.

An essential component in FoF scenarios are mobile manipulators, the combination of a mobile robot with one or more manipulators. Recent development efforts of robots manufacturers led to research platforms now available for research and the market. Examples include the youBot [2] and the omniRob platforms by KUKA, and the rob@work platform by Fraunhofer IPA depicted in Fig. 1.

Although industry views this technology as an essential component for the factory of the future, real applications are still rare. It became quickly obvious that the control concepts and algorithms developed independently for robotic arms and for mobile robots could not easily be combined and integrated. More research is necessary to exploit the capabilities of mobile manipulators in innovative applications.

In early 2012, a group of researchers concluded that the progress in mobile manipulation research could benefit significantly by organizing a competition 

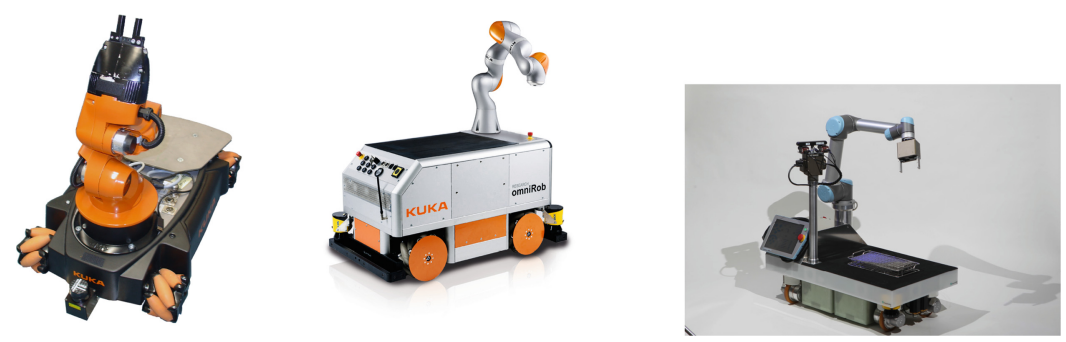

Fig. 1. Mobile manipulators from KUKA Laboratories GmbH (left) and Fraunhofer IPA (right). (Image courtesy of KUKA Laboratories GmbH and Fraunhofer IPA.)

targeted towards the factory of the future: RoboCup@Work [7]. The new competition should address research issues and appeal to both new and existing RoboCup teams who want to demonstrate their competences in a more industryrelated setting. The competition itself should be designed to combine the public appeal of robotic soccer, the competition structure of RoboCup@Home [4], and the benchmarking technology from RoboCup Rescue [5,9]. This paper describes the motivation and context of RoboCup@Work, the competition design and implementation, and the experiences made so far.

\section{Research Issues and Challenges}

RoboCup@Work targets the use of robots in work-related scenarios and tackles open research challenges in industrial and service robotics. Examples for the work-related scenarios targeted by RoboCup@Work include

- loading and unloading containers with objects of the same or different size,

- pickup or delivery of parts from/to structured or unstructured storage/heaps,

- operation of machines, including pressing buttons, opening/closing doors and drawers, and similar operations with underspecified or unknown kinematics,

- flexible planning and dynamic scheduling of production processes involving multiple agents (humans, robots, machines, tools, and parts),

- cooperative assembly of non-trivial objects with other robots or humans,

- cooperative collection of objects in large-scale spatial areas, and

- cooperative transportation of objects (robot with robots or humans).

The RoboCup@Work scenarios target difficult, still mostly unsolved problems in robotics, artificial intelligence, and advanced computer science, in particular in perception, path planning and motion planning, mobile manipulation, planning and scheduling, learning and adaptivity, and probabilistic modeling, to name just a few. Solutions to the problems posed by RoboCup@Work require sophisticated and innovative approaches and methods and their effective integration. The scenarios are defined such that the problems are sufficiently general and independent of particular industrial applications, but also sufficiently close to real application problems such that the solutions can be adapted to particular application problems with reasonable effort. 


\section{Competition Design}

The design of the RoboCup@Work competition utilizes proven ideas and concepts from other RoboCup competitions to tackle open research challenges in industrial and service robotics.

\subsection{Overview and General Ideas}

As stated previously, the new competition should combine the public appeal of robotic soccer, the competition structure of RoboCup@Home, and the benchmarking technology from RoboCup Rescue.

Being appealing to the public is not easy for a competition with industrial relevance. Using small robots instead of large industrial manipulators and keeping the competition arena small helps. Two essential ingredients for public appeal are using easy to understand (what is the robot supposed to do?) and easy to observe (is the robot doing what it is supposed to do?) tasks and realistic objects, which humans can associate with these tasks.

The competition structure of RoboCup@Home served as a model for structuring the RoboCup@Work competition. The basic element are well-defined tests, which focus on a specific task. The task can be executed by a team within a limited period of time (typically $10 \mathrm{~min}$ ). Tests may be executed repeatedly, typically on another competition day, which gives teams some room for applying bug fixes and improvements to their code or remedy hardware problems.

For scoring, RoboCup Rescue served as a role model for RoboCup@Work. We define clear objectives and performance criteria for each test and tasks the robots must perform. Scores achieved in all tests are accumulated to determine the overall ranking of teams in the competition. Furthermore, awards are given for special achievements or excellent performance on specific functionalities and tasks (best-in-class awards).

\subsection{The RoboCup@Work Competition Arena}

The environment initially designed for RoboCup@Work is depicted in Fig. 2. This design is meant only as a guiding example, as the environment should be adaptable within reasonable margins. The size of the arena is a rectangular area no less than $2 \mathrm{~m} \times 4 \mathrm{~m}$ and no more than $8 \mathrm{~m} \times 10 \mathrm{~m}$. The competition arena is partially surrounded by walls. One or more gates may be foreseen, where robots can enter or leave the arena.

The arena contains designated service areas (tagged $D_{i}$ or $S_{i}$ in the figure). Examples include loading and unloading areas, conveyor belts, rotators, storage areas, etc. Service areas may contain specific environment objects, such as racks, shelves, etc. A set of designated places is defined, which are locations in the arena that can be referred to by a unique, symbolic identifier, which are used in task specifications. The arena may foresee the use of obstacles. Obstacles may by passive or active (other robots or humans). 


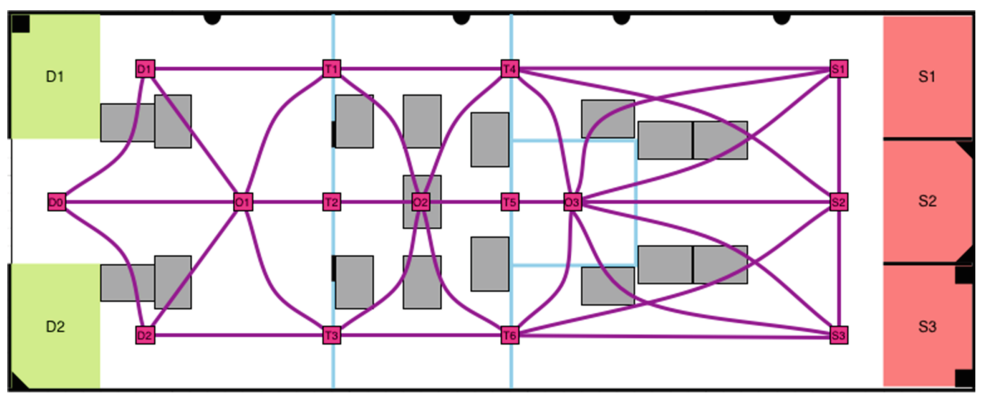

Fig. 2. Conceptual design of the RoboCup@Work competition arena.

\subsection{Manipulation Objects and Cavities Used by RoboCup@Work}

The manipulation objects used in RoboCup@Work must be objects relevant in industrial applications. An excerpt of the initial set of objects includes screws, nuts, and aluminium profiles as shown in Fig. 3. The objects are standardized DIN and ISO objects used in industry and are available worldwide. The objects pose challenging problems in terms of detection (e.g. relatively small screws) and recognition (e.g. distinguishing between nuts which differ only on the surface structure). In Fig. 4 the corresponding cavities for depositing and fitting are shown.

\subsection{BNT: Basic Navigation Test}

The first and simplest test in RoboCup@Work is the Basic Navigation Test (BNT). Teams must demonstrate their ability to safely navigate well in their environment, i.e. in a goal-oriented, autonomous, robust, and safe manner, by efficiently visiting a sequence of locations specified in a symbolic manner.

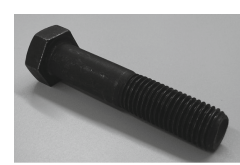

(a) M20 bolt

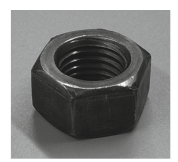

(b) M20 nut

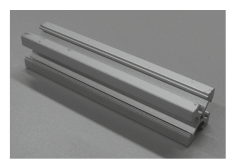

(c) alu profile

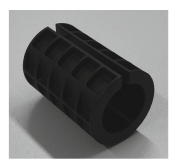

(d) R20 bushing

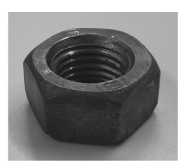

(e) M30 nut

Fig. 3. Examples of manipulation objects

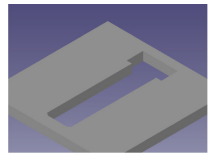

(a) M20 bolt

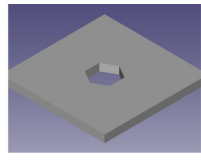

(b) M20 nut

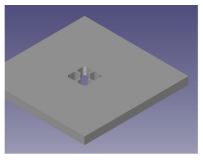

(c) alu profile

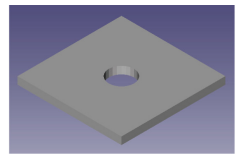

(d) R20 bushing

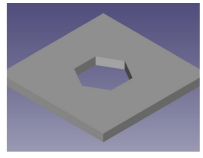

(e) M30 nut

Fig. 4. Examples of shapes for depositing/fitting manipulation objects. 
Task Description: A single robot is used. The robot is given a sequence of locations it is supposed to visit in exactly the order given by the sequence. When the test starts, the robot must request a task description from the referee box using a given protocol. The task description sent by the referee box is a string containing a sequence of triples. Each triple specifies a place, an orientation, and pause duration. Examples include

$$
\operatorname{BNT}<(S 6, N, 3),(S 2, N, 3),(D 1, S, 3),(S 5, W, 3),(D 3, E, 3),(D 4, S, 3)>
$$

and

$\operatorname{BNT}<(S 6, N, 3),(S 3, W, 3),(S 7, W, 3),(S 2, W, 3),(D 3, E, 3)>$

The robot has to move to the places specified in the task string, in the order as specified by the string, orient itself according to the orientation given, cover a place marker, pause its movement for the time in seconds as specified by the pause length, and finally leave the arena through the gate.

Scoring: The robot obtains 50 points for each successfully achieved subtask and an additional 150 points for completing the complete task specification. 20 negative points are awarded as penalty every time the robot touches an obstacle or an environment object. If two or more teams achieve the same score, time needed to achieve the equivalent result is used for tie-breaking.

\subsection{BMT: Basic Manipulation Test}

The second test is the Basic Manipulation Test (BMT). Teams must demonstrate their ability to perceive objects and perform pick and place operations with them. The focus is on the manipulation and on demonstrating safe and robust grasping and placing of objects of different size and shape.

Task Description: A single robot is used. The objective is to move a set of objects from one service area into another. The task specification consists of (i) an initial place, (ii) a source location where the objects will be found, (iii) a (nearby) destination location where the objects will have to put, (iv) a desired spatial configuration of the manipulation objects, to be produced by the robot, and (v) a final place for the robot. Manipulation objects are referenced by symbolic descriptors as defined in the rule book. Only one spatial configuration has been used so far: a line of objects. Two examples for full task specifications are:

BMT<S6, S6, S7, line (V20,R20, F20_20_B), S7>

BMT<S6, S6, S7, line (F20_20_G, F20_20_B, M20_100), S7> 


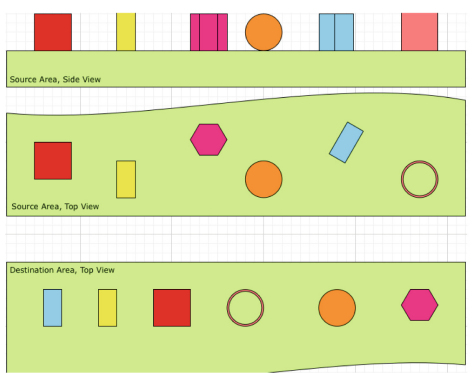

(a) Spatial configurations of objects

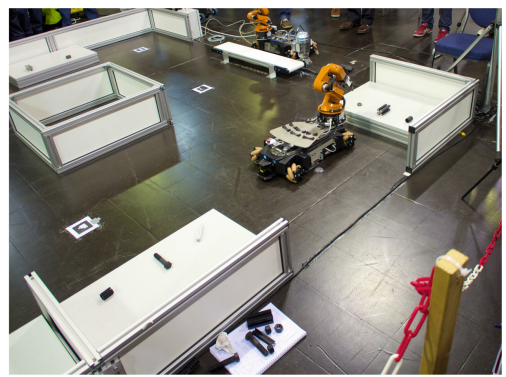

(b) Example scenario for BMT

Scoring: The robot obtains 100 points for each successfully picked-up object and another 50 points for putting it in its correct place. An additional 150 points are awarded for completing the complete task specification. If two or more teams achieve the same score, time needed to achieve the equivalent result is used for tie-breaking.

\subsection{BTT: Basic Transportation Test}

The third test is the Basic Transportation Test (BTT). Teams must demonstrate their ability to combine navigation and manipulation abilities to perform simple transportation tasks.

Task Description: A single robot is used, which is initially positioned outside of the arena near a gate to the arena. The task is to get several objects from the source service areas and to deliver them to the destination service areas. Robots may carry up to three objects simultaneously. The task specification consists of two lists: The first contains for each service area a list of manipulation objects. The second list contains for each destination service area a configuration of objects the robot is supposed to produce. Two example task specifications are:

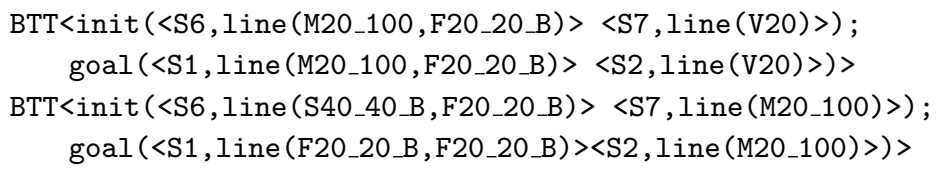

The robots can fetch objects from any source area and deliver them to destination areas in any order; they may also decide themselves whether to carry a single object or several ones simultaneously.

Scoring: The robot obtains 100 points for each successfully picked-up object and another 50 points for putting it in its correct place. An additional 150 points are awarded for completing the complete task specification. If two or more teams achieve the same score, time needed to achieve the equivalent result is used for tie-breaking. 


\subsection{PPT: Precision Placement Test}

The Precision Placement Test (PPT) was introduced in the second year of RoboCup@Work. Its purpose is to assess the robots ability to grasp and place objects into object-specific cavities. This demands advanced perception and reasoning abilities, and the capability for precise manipulation of objects.
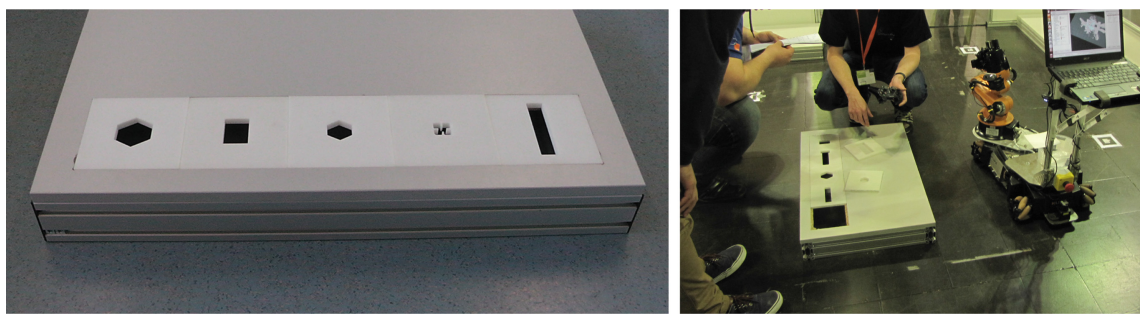

Fig. 5. Examples of cavities arrangement and environment setup.

Task Description: A single robot is placed by the team in front of the service area which stores the objects to be manipulated. The objective is to pick each object placed in the service area and place it in the corresponding cavity in the special PPT service area (see Fig. 5), which contains five cavity tiles. A PPT task specification consists of a triple which specifies the source and destination locations and a list of objects. An example is: PPT<S1, (M20, S_40_40_G), S2>, where S1 is the location where to pick up the objects M20 and S_40_40_G, and S2 designates the location where the objects need to be placed then in the corresponding cavities.

Scoring: The robot obtains 100 points for each successfully picked-up object and another 100 points for putting it in its correct cavity. A robot gets a penalty of 50 points for putting an object in the wrong cavity. An additional 200 points are awarded for completing the complete task specification. If two or more teams achieve the same score, time needed to achieve the equivalent result is used for tie-breaking.

\subsection{CBT: Conveyor Belt Test}

The Conveyor Belt Test (CBT) is another challenging test for mobile manipulators. Teams must demonstrate their ability to deal with predictable dynamics in the environment by picking or placing objects from/onto a moving conveyor belt. The test demands fast perception and manipulation skills in order to pick up objects from a operating conveyor belt. 
Task Description: A single robot is used, which is placed in some starting position outside of the arena. Initially the conveyor is switched off and three objects are already placed - with a clear distance between $5 \mathrm{~cm}$ and $10 \mathrm{~cm}$ to each other, at one end of the belt by the referee(s). The task of the robot is to navigate to the location of the conveyor belt and to grasp all three objects from the moving belt before they fall off at the other end of the belt. The robot is supposed to place the grasped objects on a suitable tray on the robot itself.

A CBT task specification simply consists of the specification of the service area where the conveyor belt is found: CBT<srv_area>.

Scoring: The robot obtains 200 points for each successfully picked-up object and another 200 points for grasping all three objects. A robot gets a penalty of 50 points for dropping an object. If two or more teams achieve the same score, time needed to achieve the equivalent result is used for tie-breaking (Fig. 6).
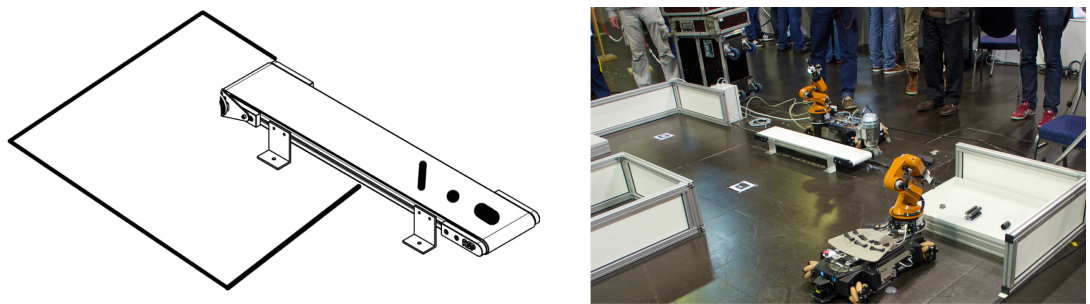

Fig. 6. Conveyor belt design (left) and example environment setup (right).

\section{Implementation: Getting the League Off the Ground}

Designing a competition is not sufficient to start a new league. It is also important to disseminate the idea and concept, to build a community of interested teams, and to develop a suitable infrastructure for organizing competition events.

Community Building: The idea of RoboCup@Work was initially developed in the context of the EU project BRICS [1], and the people driving the league development effort were mainly Ph.D. students and senior researchers working in BRICS. Two committees were created: a Technical Committee, responsible for designing the competition and developing the rule book, and an Organizing Committee for preparing demonstration events and recruiting teams. The BRICS project organized also a series of research camps between 2010 and 2012, each of which attracted about 20-25 Ph.D. students from around the world. The camps provided a great opportunity to discuss and test the interest of students and to try out some ideas that were later reused in the tests.

In May 2012, the First International RoboCup@Work Camp ${ }^{1}$ was organized at Bonn-Rhein-Sieg University (BRSU). The camp attracted 22 participants

\footnotetext{
${ }^{1}$ The camp received financial support from the RoboCup Federation via a Promoting RoboCup project. This is gratefully acknowledged.
} 
from institutes in Turkey, Germany, Austria, and Italy. Furthermore, we demonstrated the concept and organized demonstration competitions at RoboCup German Open in 2012, 2013 and 2014 (Magdeburg), RoboCup World Championships in 2012 (Mexico City) and 2013 (Eindhoven), and at IROS 2012 (Vilamoura).

League Infrastructure Development: Simultaneously with building the community, we developed the tangible league infrastructure: the competition arena, a set of parts suitable for the manipulation tasks, and a referee box for performing and controlling tests.

For 2012, we developed a low-cost, custom-built version of the initial environment as depicted in Fig. 2. In order to increase flexibility, the group at BRSU designed and built a new competition arena for 2013, based on an industrialquality aluminium profile system (see Fig. 7). This environment allows to quickly change the layout of the arena and has been used in all events since 2013. The cavities used for the Precision Placement Test were designed and 3D-printed at the mechanical workshop of the BRSU Engineering Department.

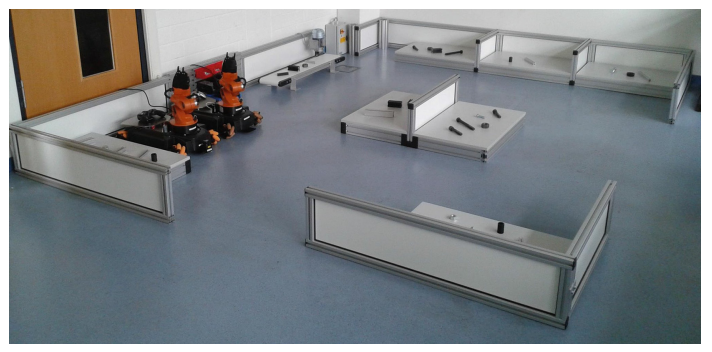

Fig. 7. The 2013 RoboCup@Work competition arena in the BRSU lab.

Selecting the set of objects for manipulation tasks was more difficult than expected. The objects should be real industrial objects, large enough to be perceivable with current perception technology, sufficiently small and light-weight so that they can be grasped and manipulated by the target robot platforms, like the KUKA youBot.

The actual execution of tests in RoboCup@Work requires a software tool that allows to define task specifications and send them to the robots. The tool also performs time-keeping operations and sends start and stop signals to the robots. An initial version of the referee box has been developed in a project course of the Master of Autonomous Systems course at BRSU, and was subsequently maintained and enhanced by the community.

\section{Participation, Performance, and Lessons Learned}

Participation and Performance: During the last two years we attracted a solid amount of teams (between 4 and 6) which regularly participate at RoboCup@Work 
competitions. So far, teams are coming from the following home countries: Netherlands, Germany, UK, Turkey, and Singapore. We are working on attracting more and more teams to make the league more sustainable. In particular, we intend to attract (see the RoboCup@Work event at IROS-2012) teams which have never participated in other RoboCup leagues, but are researching on mobile manipulation. In general, the performance of the teams increased significantly which is reported in the following.

- Basic Navigation Test: In 2012 teams moved mainly in differential-mode even though the youBot is an omni-platform and every team used available opensource software to implement navigation capabilities. Apparently it was a good idea to introduce the BNT in order to stress the importance of robust navigation for mobile manipulation. In 2014 the BNT can be considered to be solved also in the presence of dynamic obstacles.

- Basic Manipulation Test: From the very beginning we have seen diverse hardware setups effecting manipulation. Even though, so far every team participates with a KUKA youBot the mounting and placement of the manipulator on the base differs. Some teams use the default position and others placed the arm at the front. Each configuration has pros and cons concerning object reachability, field of view, and more. Further, from a perception point of view the setup differs. Some teams work solely with RGB-D cameras mounted on a sensor tower whereas others mount cameras on the arm itself. In 2012 imprecise object grasping and collisions with the environment where the normal case. In 2013 and 2014 we can report significant improvement. In particular, collisions with the environment and false-positive grasping decreased. However, some objects remain difficult to manipulate in certain object positions and teams started to replace the youBot gripper with custom-made grippers in order to grasp also bigger objects.

- Basic Transportation Test: In 2012 navigation problems obviously effected also the BTT. Every team scored very low. In 2013 and 2014 test performance improved with very few wrong placements and overall quite fast task execution.

- Precision Placement Test: We introduced the test in 2013 and almost no team prepared the test in advance. However, in 2014 we can report an improved performance. Even though, some particular objects remain advanced to be placed precisely (e.g., in the vertical configuration).

- Conveyor Belt Test: As for the PPT the CBT remains quite challenging and no teams showed real mobile manipulation (moving base and arm while the object is moving on the belt). However, as object perception and manipulation is getting faster and more reliable as seen in the BMT and BTT we strongly believe that the test will be solved soon.

Lessons Learned. Mobile manipulation remains challenging. The systematic integration of perception, planning, and control as required for the tests introduced in RoboCup@Work calls for research on various levels. In particular, we believe that for a new team the sheer amount of capabilities and competences required 
to solve the tasks can be overstraining. Hence, we recently started to share code among teams in order to learn from other solutions and also to identify best practices. As in other leagues this code releasing will be further established. Further, we would like to link software releases with educational activities such as camps as this will also help new teams to enter the league.

\section{$6 \quad$ Future Directions}

The RoboCup@Work competition has developed well so far and is considered in a stable state. Participation is increasing slowly but steadily. Some ideas for the future are expected to further increase interest and participation.

Scenario Evolution. As some of the initial tests are performed more and more robustly by the teams, the league is looking into designing more advanced tests. More interaction with automation equipment (like with the conveyor belt in the CBT test) is on the agenda. Under discussion are scenarios involving multiple robots and human-robot cooperation. Furthermore, we need to make every effort to address industrial relevance of the scenarios. Therefore, we plan to include industrial stakeholders more actively in the scenario design.

Festo Logistics League. Almost simultaneously with RoboCup@Work, Festo Didactics undertook its own initiative for establishing a league with industrial relevance. Festo adopted the concept of a manufacturing logistics scenario and, in several annual iterations, gradually tuned its competition, initially designed for a different purpose, to this idea [6]. We have been seeking cooperation with Festo from the very beginning, but due to strategic and marketing consideration Festo did not want to have their platform directly compete against other robot platforms. Nevertheless, we continue to work towards cooperation between the leagues, possibly by connecting the arenas somehow and developing scenarios where teams must control both RoboTinos, youBots and other robots.

RoCKIn Liaison. A very interesting future direction is the liaison with the EU project RoCKIn [8], where both BRSU and KUKA Labs are partners, together with three other long-time RoboCup researchers. The RoCKIn project has developed its own versions of the RoboCup@Home and RoboCup@Work competitions, with special attention to benchmarking and networked robotics. It is a clear objective that the RoCKIn@Work ideas and concepts will be assimilated by RoboCup@Work in the near future.

EuROC Liaison. Last but not least, we would like to point to the EuROC European Robotics Challenge [3], an EU-funded project started in 2014. EuROC defines three challenges, two of which have picked up ideas proposed by RoboCup@Work: the Reconfigurable Interactive Manufacturing Cell (RIMC) challenge and the Shop Floor Logistics and Manipulation (SFLM) challenge. To some extent, this effort can already be viewed as an impact of the RoboCup@Work effort. 


\section{Conclusion}

This paper presents the ideas and concepts behind the RoboCup@Work Demonstration League. We have discussed, motivation, research challenges, competition design and implementation in detail and reported about the development of the league, lessons learned, and future directions. The conclusion is that RoboCup@Work is a timely and well-defined competition with high industrial relevance, and a very interesting extension of the spectrum of RoboCup leagues.

\section{References}

1. Bischoff, R., Guhl, T., Prassler, E., Nowak, W., Kraetzschmar, G., Bruyninckx, H., Soetens, P., Haegele, M., Pott, A., Breedveld, P., Broenink, J., Brugali, D., Tomatis, N.: BRICS - best practice in robotics. In: 41st International Symposium on Robotics (ISR) and 6th German Conference on Robotics (ROBOTIK), pp. 1-8, June 2010

2. Bischoff, R., Huggenberger, U., Prassler, E.: Kuka youbot - a mobile manipulator for research and education. In: IEEE International Conference on Robotics and Automation (2011)

3. EuRoC Project: European robotics challenges (2014). http://www.euroc-project. $\mathrm{eu} /$

4. Holz, D., Iocchi, L., van der Zant, T.: Benchmarking intelligent service robots through scientific competitions: the RoboCup@Home approach. In: Proceedings of the AAAI Spring Symposium Designing Intelligent Robots: Reintegrating AI II (2013)

5. Kitano, H., Tadokoro, S.: RoboCup rescue - a grand challenge for multiagent and intelligent systems. AI Mag. 22, 39-52 (2001)

6. Niemueller, T., Ewert, D., Reuter, S., Ferrein, A., Jeschke, S., Lakemeyer, G.: RoboCup logistics league sponsored by Festo: a competitive factory automation testbed. In: Behnke, S., Veloso, M., Visser, A., Xiong, R. (eds.) RoboCup 2013. LNCS, vol. 8371, pp. 336-347. Springer, Heidelberg (2014)

7. RoboCup@Work: RoboCup@Work (2014). http://www.robocupatwork.org

8. RoCKIn: Rockin (2014). http://www.rockinrobotchallenge.eu/

9. Sheh, R., Kimura, T., Mihankhah, E., Pellenz, J., Schwertfeger, S., Suthakorn, J.: The RoboCupRescue robot league: guiding robots towards fieldable capabilities. In: IEEE Workshop on Advanced Robotics and its Social Impacts (ARSO), pp. 31-34, Oct 2011

10. TAPAS Project: Robotics-enabled logistics and assistive services for the transformable factory of the future (tapas) (2013). http://www.tapas-project.eu/ 